Europhysics Letters

PREPRINT

\title{
Sympathetic cooling of trapped fermions by bosons in the presence of particle losses
}

\author{
Z. IDZiAsZeK ${ }^{1,2}$, L. SAntos ${ }^{3}$ and M. Lewenstein ${ }^{4}$ \\ 1 Istituto Nazionale per la Fisica della Materia, BEC-INFM Trento, I-38050 Povo \\ $(T N)$, Italy \\ 2 Centrum Fizyki Teoretycznej, Polska Akademia Nauk, 02-668 Warsaw, Poland \\ 3 Institut für Theoretische Physik III, Universität Stuttgart, Pfaffenwaldring 57 V, D- \\ 70550 Stuttgart, Germany \\ 4 Institut für Theoretische Physik, Universität Hannover, D-30167 Hannover, Germany
}

PACS. 03.75.Ss - Degenerate Fermi gases.

\begin{abstract}
We study the sympathetic cooling of a trapped Fermi gas interacting with an ideal Bose gas below the critical temperature of the Bose-Einstein condensation. We derive the quantum master equation, which describes the dynamics of the fermionic component, and postulating the thermal distribution for both gases we calculate analytically the rate at which fermions are cooled by the bosonic atoms. The particle losses constitute an important source of heating of the degenerate Fermi gas. We evaluate the rate of loss-induced heating and derive analytical results for the final temperature of fermions, which is limited in the presence of particle losses.
\end{abstract}

Evaporative cooling has proven to be an essential tool to obtain degenerate Fermi gases [1-6]. In Fermi systems, the $s$-wave collisions of indistinguishable particles are forbidden due to the antisymmetry requirement, and therefore the only way to cool fermions using collisions, is to cool them sympathetically by bringing them in contact with atoms in different hyperfine states, or another species. In the regime of quantum degeneracy, however, also the collisional processes in Fermi-Fermi mixtures are strongly suppressed due to Pauli blocking. As a consequence, the efficiency of sympathetic cooling is reduced, and the particle losses, which provide an important source of heating in the degenerate regime [7], prevent from reaching the lower temperatures T, necessary, for instance, for achievement of the superfluid BCS state. This problem may be avoided by adiabatic crossing of a Feshbach resonance, since very low temperatures may be achieved [8] when moving from a molecular BEC regime [9] to the BCS regime. In this crossover, which has recently attracted a large attention, strong evidences for superfluidity have been observed [10-14]. Another obstacle appears when a Fermionic gas is sympathetically cooled using a Bose-Einstein condensate, since as a consequence of the superfluid properties of the condensate, the sympathetic cooling is limited to velocities larger than the sound velocity [15].

Sympathetic cooling has been recently considered by several theoretical groups [15-22]. It should be stressed, however, that the limitations due to particle losses have been addressed

(C) EDP Sciences 
in detail only recently by Carr et al. [23], who have studied the cooling of fermions by an ideal homogeneous Bose gas at $T=0$. In this paper we extend the result of Ref. [23], and investigate the influence of the particle losses on the sympathetic cooling of harmonically trapped gases at finite $T$. We consider here a simplified model which assumes that fermions are in contact with an ideal Bose gas, which is kept at constant $T$. This can be achieved if the bosons are continuously cooled by means of evaporative cooling or eventually when the bosonic component is much larger than the fermionic one.

The gas of $N_{f}$ fermions is confined in a harmonic trap of frequency $\omega_{f}$. In the following we assume that the trapping potential is spherically symmetric, however, our results can be generalized to anisotropic traps. The fermions interact with a gas of $N_{b}$ bosonic atoms, confined in a harmonic trap of frequency $\omega_{b}$. We treat the gas of bosons as a reservoir, which assumes that its temperature $T_{b}$ and statistical properties do not change during the cooling process. The Hamiltonian of the system reads

$$
H=\sum_{\mathbf{n}} \varepsilon_{\mathbf{n}} a_{\mathbf{n}}^{\dagger} a_{\mathbf{n}}+\sum_{\mathbf{m}} \varepsilon_{\mathbf{m}}^{b} b_{\mathbf{m}}^{\dagger} b_{\mathbf{m}}+\sum_{\mathbf{n}, \mathbf{n}^{\prime}, \mathbf{m}, \mathbf{m}^{\prime}} U_{\mathbf{n}, \mathbf{n}^{\prime}, \mathbf{m}, \mathbf{m}^{\prime}} a_{\mathbf{n}}^{\dagger} a_{\mathbf{n}^{\prime}} b_{\mathbf{m}}^{\dagger} b_{\mathbf{m}^{\prime}}
$$

where $\mathbf{n}$ and $\mathbf{m}$ label the states of the three-dimensional harmonic oscillator, $\varepsilon_{\mathbf{n}}$ and $\varepsilon_{\mathbf{m}}^{b}$ denote single particle energies of fermions and bosons, respectively, and $a_{\mathbf{n}}\left(b_{\mathbf{m}}\right)$ is the creation operator of fermions (bosons) fulfilling usual anticommutation (commutation) relations. In the low-temperature regime considered here, the scattering between atoms of both species is purely of $s$-wave type and can be modeled by a delta pseudopotential with scattering length $a$. Thus the matrix elements of the boson-fermion interactions are given by

$$
U_{\mathbf{n}, \mathbf{n}^{\prime}, \mathbf{m}, \mathbf{m}^{\prime}}=g \int \mathrm{d}^{3} r \psi_{\mathbf{n}}^{*}(\mathbf{r}) \psi_{\mathbf{n}^{\prime}}(\mathbf{r}) \phi_{\mathbf{m}}^{*}(\mathbf{r}) \phi_{\mathbf{m}^{\prime}}(\mathbf{r})
$$

where $\psi_{\mathbf{n}}(\mathbf{r})$ and $\phi_{\mathbf{m}}(\mathbf{r})$ are the single-particle eigenfunctions of fermions and bosons, respectively, $g=2 \pi \hbar^{2} a / \mu_{m}$, and $\mu_{m}=m_{b} m_{f} /\left(m_{b}+m_{f}\right)$ denotes the reduced mass $\left(m_{b}\right.$ is the mass of boson and $m_{f}$ is the mass of fermion). We apply standard techniques of the theory of quantum-stochastic processes [24,25] and obtain the quantum Master equation (ME) for the density matrix $\rho$ of the fermions [16]

$\frac{d \rho}{d t}=-\frac{i}{\hbar}\left[H_{F}+H_{F-F}, \rho\right]+\sum_{\mathbf{n}, \mathbf{n}^{\prime}, \mathbf{m}, \mathbf{m}^{\prime}} \Gamma_{\mathbf{n}, \mathbf{n}^{\prime}}^{\mathbf{m}, \mathbf{m}^{\prime}}\left(2 a_{\mathbf{m}}^{\dagger} a_{\mathbf{m}^{\prime}} \rho a_{\mathbf{n}}^{\dagger} a_{\mathbf{n}^{\prime}}-a_{\mathbf{n}}^{\dagger} a_{\mathbf{n}^{\prime}} a_{\mathbf{m}}^{\dagger} a_{\mathbf{m}^{\prime}} \rho-\rho a_{\mathbf{n}}^{\dagger} a_{\mathbf{n}^{\prime}} a_{\mathbf{m}}^{\dagger} a_{\mathbf{m}^{\prime}}\right)$

where $H_{F}=\sum_{\mathbf{n}} \varepsilon_{\mathbf{n}} a_{\mathbf{n}}^{\dagger} a_{\mathbf{n}}$ and the rate coefficients are of the form:

$$
\Gamma_{\mathbf{n}, \mathbf{n}^{\prime}}^{\mathbf{m}, \mathbf{m}^{\prime}}=\frac{\pi}{\hbar^{2} \omega_{b}} \delta\left(\varepsilon_{\mathbf{n}}+\varepsilon_{\mathbf{m}}, \varepsilon_{\mathbf{n}^{\prime}}+\varepsilon_{\mathbf{m}^{\prime}}\right) \sum_{\mathbf{k}, \mathbf{q}} U_{\mathbf{n}, \mathbf{n}^{\prime}, \mathbf{q}, \mathbf{k}} U_{\mathbf{m}, \mathbf{m}^{\prime}, \mathbf{k}, \mathbf{q}} n_{\mathbf{q}}^{b}\left(1+n_{\mathbf{k}}^{b}\right) \delta\left(\varepsilon_{\mathbf{m}}+\varepsilon_{\mathbf{k}}^{b}, \varepsilon_{\mathbf{m}^{\prime}}+\varepsilon_{\mathbf{q}}^{b}\right) .
$$

In Eq. (4), $\delta\left(\varepsilon, \varepsilon^{\prime}\right)$ denotes the Kronecker delta function, which accounts for the conservation of energy during collisions between fermions and bosons, and $n_{\mathbf{n}}^{b}$ denote the occupation numbers of the bosonic reservoir. The operator $H_{F-F}$ describes an effective interaction between fermions, which is mediated by bosonic atoms:

$$
H_{F-F}=\sum_{\mathbf{n}, \mathbf{n}^{\prime}, \mathbf{m}, \mathbf{m}^{\prime}} \Delta_{\mathbf{n}, \mathbf{n}^{\prime}}^{\mathbf{m}, \mathbf{m}^{\prime}} a_{\mathbf{n}}^{\dagger} a_{\mathbf{n}^{\prime}} a_{\mathbf{m}}^{\dagger} a_{\mathbf{m}^{\prime}}
$$

where

$$
\Delta_{\mathbf{n}, \mathbf{n}^{\prime}}^{\mathbf{m}, \mathbf{m}^{\prime}}=\delta\left(\varepsilon_{\mathbf{n}}+\varepsilon_{\mathbf{m}}, \varepsilon_{\mathbf{n}^{\prime}}+\varepsilon_{\mathbf{m}^{\prime}}\right) \sum_{\mathbf{k}, \mathbf{q}} U_{\mathbf{n}, \mathbf{n}^{\prime}, \mathbf{q}, \mathbf{k}} U_{\mathbf{m}, \mathbf{m}^{\prime}, \mathbf{k}, \mathbf{q}} n_{\mathbf{q}}^{b}\left(1+n_{\mathbf{k}}^{b}\right) \frac{1-\delta\left(\varepsilon_{\mathbf{m}}+\varepsilon_{\mathbf{k}}^{b}, \varepsilon_{\mathbf{m}^{\prime}}+\varepsilon_{\mathbf{q}}^{b}\right)}{\varepsilon_{\mathbf{m}^{\prime}}+\varepsilon_{\mathbf{q}}^{b}-\varepsilon_{\mathbf{m}}-\varepsilon_{\mathbf{k}}^{b}} .
$$


We assume that interactions between bosons and fermions are sufficiently small, and hence $H_{F-F}$ does not influence significantly the single-particle eigenstates in a harmonic trap.

To calculate the dynamics of a fermionic gas from the ME we introduce some further approximations. First, by considering the decoherence induced by the interactions between bosons and fermions one can argue that the nondiagonal elements of the density matrix in the energy representation decay on a time-scale much shorter than the time scale of the dynamics due to sympathetic cooling [20]. Inclusion of $H_{F-F}$ lifts the degeneracy of the states of equal energy but different occupation numbers of single-particle eigenstates, and hence the decoherence affects also the nondiagonal terms with respect to the occupation numbers. Then, we apply ergodic approximation assuming that for a given energy all diagonal elements of $\rho$ are equal. This requires that the process of equilibration within a single energy shell is much faster than for the whole system. Thus, the density matrix $\rho$ can be written in the form [20]

$$
\rho(t)=\sum_{E, \lambda} \frac{p_{E}(t)}{\Gamma\left(N_{f}, E\right)}\left|E,\left\{n_{\mathbf{n}}\right\}_{\lambda}\right\rangle\left\langle E,\left\{n_{\mathbf{n}}\right\}_{\lambda}\right|
$$

Here, $\lambda$ enumerates microstates $\left\{n_{\mathbf{n}}\right\}_{\lambda}$ of the fermionic component, and $\left|E,\left\{n_{\mathbf{n}}\right\}_{\lambda}\right\rangle$ represents the state with energy $E$ and a given distribution of fermions. The probability that the system of fermions has energy $E$ is denoted by $p_{E}(t)$, while $\Gamma\left(N_{f}, E\right)$ is the number of microstates with energy $E$ and number of particles $N_{f}$. Substituting $\rho(t)$ given by Eq. (17) into the ME (3), and neglecting the terms due to the $H_{F-F}$, we obtain the equations determining the dynamics of the probabilities $p_{E}(t)$ :

$$
\frac{d p_{E}}{d t}=2 \sum_{\mathbf{n}, \mathbf{l}} \Gamma_{\mathbf{n}, \mathbf{m}}^{\mathbf{m}, \mathbf{n}}\left(p_{E+\varepsilon_{\mathbf{n}}-\varepsilon_{\mathbf{m}}}(t)\left\langle n_{\mathbf{n}}\left(1-n_{\mathbf{m}}\right)\right\rangle_{E+\varepsilon_{\mathbf{n}}-\varepsilon_{\mathbf{m}}}-p_{E}(t)\left\langle n_{\mathbf{n}}\left(1-n_{\mathbf{m}}\right)\right\rangle_{E}\right),
$$

where $\left\langle n_{\mathbf{n}}\left(1-n_{\mathbf{m}}\right)\right\rangle_{E}$ stands for the microcanonical average over microstates with energy $E$, and $n_{\mathbf{n}}=a_{\mathbf{n}}^{\dagger} a_{\mathbf{n}}$. Probabilities $p_{E}(t)$ determine all the thermodynamic quantities of the gas of fermions. In particular, its mean energy is given by $\langle E\rangle=\sum_{E} E p_{E}(t)$. Combining this expression with Eq. (8), we calculate the rate $\Gamma \equiv-\frac{d}{d t}\langle E\rangle$ of cooling of the fermions by the bosonic reservoir:

$$
\Gamma=2 \sum_{\mathbf{n}, \mathbf{m}} \Gamma_{\mathbf{n}, \mathbf{m}}^{\mathbf{m}, \mathbf{n}}\left(\varepsilon_{\mathbf{n}}-\varepsilon_{\mathbf{m}}\right) \sum_{E} p_{E}(t)\left\langle n_{\mathbf{n}}\left(1-n_{\mathbf{m}}\right)\right\rangle_{E} .
$$

Since we are mainly interested in the regime where fermions are sufficiently cold and the effects of heating due to the losses become important, we assume that the gas of fermions is close to equilibrium and evaluate $p_{E}(t)$ from the canonical or the grand-canonical ensemble. We chose the latter possibility and postulate the grand-canonical distribution for both fermionic component and bosonic reservoir. Substituting Eq. (4) for the rates $\Gamma_{\mathbf{n}, \mathbf{m}}^{\mathbf{m}}$ into Eq. (9), we arrive at

$$
\Gamma=\frac{2 \pi}{\hbar^{2} \omega_{b}} \sum_{\mathbf{n}, \mathbf{m}, \mathbf{k}, \mathbf{q}}\left|U_{\mathbf{m}, \mathbf{n}, \mathbf{k}, \mathbf{q}}\right|^{2} n_{\mathbf{n}}\left(1-n_{\mathbf{m}}\right) n_{\mathbf{q}}^{b}\left(1+n_{\mathbf{k}}^{b}\right)\left(\varepsilon_{\mathbf{n}}-\varepsilon_{\mathbf{m}}\right) \delta\left(\varepsilon_{\mathbf{n}}+\varepsilon_{\mathbf{q}}, \varepsilon_{\mathbf{m}}+\varepsilon_{\mathbf{k}}\right)
$$

where $n_{\mathbf{n}}=z e^{-\beta \varepsilon_{\mathbf{n}}} /\left(1+z e^{-\beta \varepsilon_{\mathbf{n}}}\right), n_{\mathbf{q}}^{b}=z_{b} e^{-\beta_{b} \varepsilon_{\mathbf{q}}^{b}} /\left(1-z_{b} e^{-\beta_{b} \varepsilon_{\mathbf{q}}^{b}}\right), \beta=1 /\left(k_{B} T\right), \beta_{b}=1 /\left(k_{B} T_{b}\right)$, and $z, z_{b}$ denote the fugacities of fermions and bosons respectively. First we perform summation over degeneracies of the single-particle states. To this end we utilize the following approximate result

$$
\sum_{\mathbf{n}, \mathbf{m}, \mathbf{k}, \mathbf{q}}\left|U_{\mathbf{m}, \mathbf{n}, \mathbf{k}, \mathbf{q}}\right|^{2} \delta\left(\varepsilon_{\mathbf{n}}, e_{1}\right) \delta\left(\varepsilon_{\mathbf{m}}, e_{2}\right) \delta\left(\varepsilon_{\mathbf{q}}, e_{3}\right) \delta\left(\varepsilon_{\mathbf{k}}, e_{4}\right) \approx \frac{D\left(\min \left(e_{3}, e_{4}\right)\right) g^{2}}{4 \pi^{4} a_{b}^{2} a_{f}^{4}}
$$


where $a_{b}=\sqrt{\hbar / m_{b} \omega_{b}}, a_{f}=\sqrt{\hbar / m_{f} \omega_{f}}, D(e)$ denote the degeneracy of the energy shell $e$, and $\min \left(e_{3}, e_{4}\right)$ represents the minimum of $e_{3}$ and $e_{4}$. This formula can be derived by taking the semiclassical limit of Eq. (10) and comparing with the semiclassical quantum Boltzmann equation in ergodic approximation [21,26,27]. The summation formula is valid for $\min \left(e_{3}, e_{4}\right) /\left(\hbar \omega_{b}\right) \leq \lambda^{2} \min \left(e_{1}, e_{2}\right) /\left(\hbar \omega_{f}\right)$ and $\min \left(e_{3}, e_{4}\right) /\left(\hbar \omega_{b}\right) \leq \lambda^{-2} \min \left(e_{1}, e_{2}\right) /\left(\hbar \omega_{f}\right)$ where $\lambda=a_{f} / a_{b}$. For values of $\lambda$ which are not much larger or much smaller than one, this condition is fulfilled for collisions at sufficiently low temperatures, which take place close to the Fermi surface for fermions and close to the ground state for bosons. We have confirmed numerically the validity of formula (11), and verified that it is quite accurate even for the lowest states of the harmonic potential. Substituting (11) into Eq. (10) yields

$$
\Gamma=\frac{2 A \omega_{b}}{\pi} \sum_{e_{1}, e_{2}, e_{3}, e_{4}} n_{e_{1}}\left(1-n_{e_{2}}\right) n_{e_{3}}^{b}\left(1+n_{e_{4}}^{b}\right)\left(e_{1}-e_{2}\right) D\left(\min \left(e_{3}, e_{4}\right)\right) \delta\left(e_{1}+e_{3}, e_{2}+e_{4}\right),
$$

where $n_{e_{1}}=z e^{-\beta e_{1}} /\left(1+z e^{-\beta e_{1}}\right)$ and $n_{e_{3}}^{b}=z_{b} e^{-\beta_{b} e_{3}} /\left(1-z_{b} e^{-\beta_{b} e_{3}}\right)$, and $A=m_{b}^{2} a^{2} a_{b}^{2} /\left(\mu_{m}^{2} a_{f}^{4}\right)$. For a sufficiently large system, the summation over $e_{1}, e_{2}, e_{3}, e_{4}$ can be replaced by an integration, which corresponds to taking the thermodynamic limit. This procedure is correct with respect to the collisions with the thermal part of the bosonic component. In the case of a Bose-condensed reservoir, the single-particle ground state requires a separate treatment. Let us first consider the rate $\Gamma_{\text {th }}$ due to the interactions with the thermal atoms in the reservoir. Replacing sums by integrals, after some algebra we obtain

$$
\Gamma_{\mathrm{th}}=\frac{4 A}{\pi} \frac{\left(k_{B} T\right)^{3}\left(k_{B} T_{b}\right)^{3}}{\hbar^{5} \omega_{f}^{2} \omega_{b}^{2}} \sum_{l=0}^{\infty} \zeta(3,1+l)\left[\zeta\left(3, \frac{T}{T_{b}} l+1\right)-\zeta\left(3, \frac{T}{T_{b}}(1+l)\right)\right]
$$

which is valid for temperatures of fermions much smaller than the Fermi temperature $T_{F}$, and under assumption that the reservoir is Bose-condensed. Here $\zeta(s, a)$ denotes the Hurwitz zeta function [28]. We note that $\Gamma_{\text {th }}$ does not depend on the number of fermions. This is is related with the fact that at low temperatures the collisions occur only close to the Fermi surface. On the contrary, taking into account that the number of the thermal atoms in a trapped Bose-condensed gas is $N_{\mathrm{th}}=\zeta(3)\left(k_{B} T_{b} /\left(\hbar \omega_{b}\right)\right)^{3}$, we observe that $\Gamma_{\text {th }}$ is proportional to $N_{\mathrm{th}}$. When the temperature of both components is equal, we expect that there will be no energy exchange between bosons and fermions and, as can be easily verified, this feature is properly taken into account by Eq. (13). To calculate the rate $\Gamma_{0}$ describing the cooling due to collisions with the condensed part of the reservoir, we replace sums by integrals in Eq. (10), putting $e_{3}=0$ or $e_{4}=0$. This yields

$$
\Gamma_{0}=\frac{4 \zeta(3) A}{\pi} \frac{N_{0} \omega_{b}}{\left(\hbar \omega_{f}\right)^{2}}\left[\left(k_{B} T\right)^{3}-\left(k_{B} T_{b}\right)^{3}\right] .
$$

We note that at low temperatures the energy exchange with the condensate is proportional to the number of condensed atoms $N_{0}$, but does not depend on the number of fermions.

We turn now to the description of the heating due to particle losses in the degenerate Fermi gas. The losses create holes in the Fermi sea, which after subsequent thermalization increase the temperature of the system [7]. We assume that particles are removed with a rate $\gamma$, which is the same for all single-particles states: $\frac{d}{d t}\left\langle n_{\mathbf{n}}\right\rangle=-\gamma\left\langle n_{\mathbf{n}}\right\rangle$, where $\left\langle n_{\mathbf{n}}\right\rangle$ denotes the mean occupation number of the state $\mathbf{n}$. For background losses, the rate $\gamma$ does not depend on the density of fermions $n_{f}$, while in the case of two- and three-body losses it is proportional to $n_{f}$ and $n_{f}^{2}$, respectively. The removal process does not change the mean energy per particle: $\left(\langle E\rangle / N_{f}\right)(t)=$ const. At temperatures $T / T_{F} \ll 1,\langle E\rangle / N_{f}=$ 
$3 E_{F}\left(N_{f}\right) / 4+\left(\pi^{2} / 2\right) k_{B}^{2} T^{2} / E_{F}\left(N_{f}\right)$ with the Fermi energy $E_{F}\left(N_{f}\right) \approx \hbar \omega_{f}\left(6 N_{f}\right)^{1 / 3}$. The creation of holes, can be equivalently described as a process that heats the system, but, in first approximation, does not change the number of particles. We obtain that the rate of heating is given by

$$
\Gamma_{\mathrm{H}}=N_{f} \gamma E_{F}\left[\frac{1}{4}-\frac{\pi^{2}}{6}\left(T / T_{F}\right)^{2}\right],
$$

where we neglect terms of order higher than $\left(T / T_{F}\right)^{2}$.

In the absence of particle losses, the Fermi component is cooled down until it reaches the temperature of the bosonic reservoir $T_{b}$. The heating produced by the hole creation, however, prevents from reaching $T_{b}$, and sets a constraint on the lowest temperature achievable with the sympathetic colling. The final temperature of the Fermi gas $T_{\text {fin }}$, at which the cooling is balanced by the hole heating, is determined by the condition: $\Gamma_{\mathrm{th}}+\Gamma_{0}=\Gamma_{\mathrm{H}}$. When the losses are sufficiently small, the final temperature of fermions $T_{\text {fin }}$ only slightly differs from $T_{b}$, and in this case its value can be estimated analytically. To this end, we perform a Taylor expansion of the cooling rate $\Gamma=\Gamma_{\text {th }}+\Gamma_{0}$ at $T=T_{B}$, keeping only the lowest order term $\left.\Gamma(T) \approx(\partial \Gamma /(\partial T))\right|_{T=T_{b}}\left(T-T_{b}\right)$. This yields

$$
\Gamma \approx \frac{12 A}{\pi} \frac{\omega_{b}\left(k_{B} T_{b}\right)^{3}}{\left(\hbar \omega_{f}\right)^{2}} \frac{T-T_{b}}{T_{b}}\left[\zeta(3) N_{0}+c\left(\frac{k_{B} T_{b}}{\hbar \omega_{b}}\right)^{3}\right]
$$

which is valid for $\left|T-T_{b}\right| \ll T_{b}$. Here $c$ is a numerical constant given by $c=\sum_{l=0}^{\infty} \zeta(3,1+$ $l) \zeta(4,1+l) \simeq 1.3196$. For temperatures of the bosonic reservoir $T_{b}$ much smaller than the critical temperature $T_{C}$, one can neglect $c\left(k_{B} T_{b} /\left(\hbar \omega_{b}\right)\right)^{3}$ in comparison to $\zeta(3) N_{0}$, and in this regime Eq. (16) reduces to

$$
\Gamma \approx \frac{12 A}{\pi} \frac{\hbar \omega_{b}^{4}}{\omega_{f}^{2}}\left(\frac{T_{b}}{T_{C}}\right)^{3} \frac{T-T_{b}}{T_{b}} N_{0}^{2} .
$$

This formula combined with the rate $\Gamma_{\mathrm{H}}$, which at $T \ll T_{F}$ can be approximated by $\Gamma_{\mathrm{H}} \approx$ $N_{f} \gamma E_{F} / 4$, leads to the following result for the final temperature of the Fermi gas

$$
T_{\text {fin }}=T_{b}\left(1+\frac{6^{1 / 3} \pi}{48 A} \frac{\gamma \omega_{f}^{3}}{\omega_{b}^{4}} \frac{N_{f}^{4 / 3}}{N_{0}^{2}}\left(\frac{T_{C}}{T_{b}}\right)^{3}\right) .
$$

The lowest temperature in the sympathetic cooling of a Fermi gas is achieved when the reservoir is totally condensed. In this particular case, the final temperature can be also calculated analytically. We observe that at $T_{b}=0, \Gamma_{\text {th }}=0$, while for $T \ll T_{F}$ the rate of lossinduced heating can be approximated by $\Gamma_{\mathrm{H}} \approx N_{f} \gamma E_{F} / 4$. This yields the final temperature of fermions:

$$
T_{\text {fin }}=b T_{F}\left(\frac{\gamma N_{f}^{1 / 3}}{A \omega_{b} N_{0}}\right)^{1 / 3}
$$

where $b=\left(6^{1 / 3} \pi /(96 \zeta(3))\right)^{1 / 3} \simeq 0.367$. We note that $T_{\text {fin }}$ very weakly depends on the number of fermions $N_{f}$, and is mainly determined by the efficiency of the cooling process (through parameters $N_{0}, A, \omega_{b}$ ), and by the loss rate $\gamma$.

To estimate the influence of the loss-induced heating on the final temperature of fermions, we have performed numerical calculations for the mixture of bosonic ${ }^{7} \mathrm{Li}$ with fermionic ${ }^{6} \mathrm{Li}$ $[2,3]$. In our calculations we assume: $N_{b}=1.5 \times 10^{4}, N_{f}=4 \times 10^{3}, \omega_{b}=\omega_{f}=2 \pi \times 1270 \mathrm{~Hz}$, 


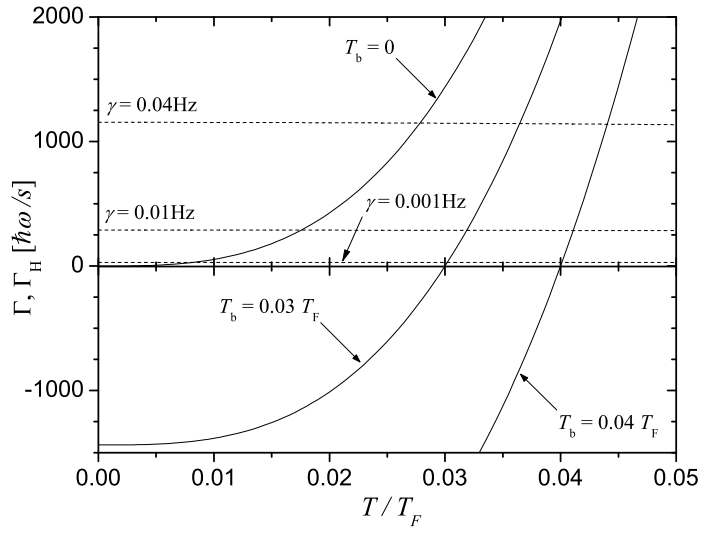

Fig. 1

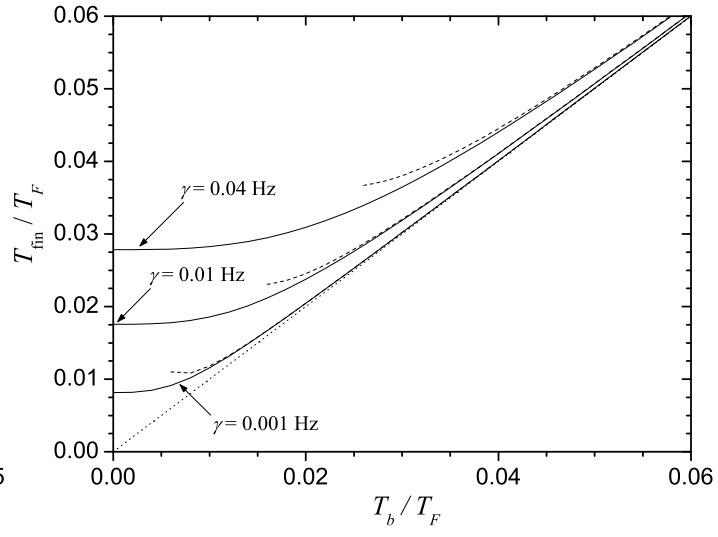

Fig. 2

Fig. 1 - Cooling rates $\Gamma$ (solid lines) and heating rates $\Gamma_{\mathrm{H}}$ (dashed lines) versus the fermion temperature $T$. The rates were calculated for three different temperatures of the bosonic reservoir: $T_{b}=0$, $0.03 T_{F}, 0.04 T_{F}$, and for three different loss rates $\gamma=0.04 \mathrm{~Hz}, 0.01 \mathrm{~Hz}$, and $0.001 \mathrm{~Hz}$. The values of the other parameters correspond to the experiment described in [2] (see text for details).

Fig. 2 - Final temperature of the Fermi gas versus the temperature of the bosonic reservoir $T_{b}$ calculated for the parameters of Fig. 1 The plot shows the numerical values evaluated from the rates given by Eqs. (13), (14), and (15) (solid lines), the approximate result of Eq. (18) (dashed lines), and the line $T=T_{b}$, which is the final temperature in the absence of losses (dotted line).

and $a=2.0 \mathrm{~nm}$, which corresponds to the experimental parameters of Ref. [2]. Fig. [1]shows, as a function of the temperature of the fermions, the cooling rates $\Gamma=\Gamma_{0}+\Gamma_{\text {th }}$ evaluated from Eqs. (13) and (14) for three different temperatures of the bosons: $T_{b}=0,0.03 T_{F}$, and $0.04 T_{F}$ (solid lines). The dashed lines show the heating rate $\Gamma_{\mathrm{H}}$ given by Eq. (15). Since the loss rate $\gamma$ depends on the particular experimental conditions, we have used three different values of $\gamma$ : $0.04 \mathrm{~Hz}, 0.01 \mathrm{~Hz}$ and $0.001 \mathrm{~Hz}$. The final temperature of fermions is determined by the crossing of $\Gamma$ and $\Gamma_{\mathrm{H}}$. We observe that for the totally condensed reservoir $\left(T_{b}=0\right)$ and for fermion temperatures $T \lesssim 0.01 T_{C}$, the cooling rates become very small due to Pauli blocking. In this regime the presence of even very small losses prevents from reaching lower temperatures. For higher temperatures of the bosonic component $\left(T_{b}=0.03 T_{F}\right.$ and $\left.T_{b}=0.04 T_{F}\right)$, the hole heating shifts only slightly the final fermion temperature with respect to $T_{b}$, and $T_{\text {fin }}$ can be determined by expanding the cooling rate around $T_{b}$ as in the case of Eq. (18). The dependence of the final fermion temperature $T_{\text {fin }}$ on $T_{b}$ is shown in Fig. 2 where the results calculated from the condition $\Gamma_{\mathrm{th}}+\Gamma_{0}=\Gamma_{\mathrm{H}}$, using Eqs. (13), (14) and (15), are compared with Eq. (18).

In our analysis we have neglected the interactions within the bosonic reservoir. This can be a good approximation for the conditions of experiment [2], where the condensate is relatively small $\left(N_{b} \sim 10^{4}\right)$ and the scattering length for ${ }^{7} \mathrm{Li}$ atoms in the state $\left|F=1, m_{F}=-1\right\rangle$ is $a=0.27 \mathrm{~nm}$. In general, however, the interactions modify the density profile of the bosonic cloud, and introduce the quantum depletion of the condensate. For repulsive forces the size of an interacting condensate is much larger in comparison to the ideal-gas. This can reduce the collisional rate between bosons and fermions and consequently decrease the cooling efficiency. On the other hand, the quantum depletion reduces the number of condensed atoms, transfering them into excited states, which increases the effective temperature of the reservoir. Therefore, 
the most effective cooling is achieved for noninteracting bosons, which can be eventually realized with the help of Feshbach resonances.

Summarizing, we have investigated the sympathetic cooling of a trapped Fermi gas in contact with a Bose-condensed reservoir, and shown that the loss-induced heating limits the cooling at low temperatures. In the regime of weak heating and in the case of totally condensed Bose gas, we have derived the analytical results for the final temperature of the fermions.

We acknowledge fruitful discussions with L. Carr. Z.I. acknowledges BEC-INFM for hospitality and financial support. This work has been supported by the Deutsche Forschungsgemeinschaft (SFB 407, SPP 1116, 432 POL), ESF Program BEC2000+, and by the Alexander von Humboldt foundation.

\section{REFERENCES}

[1] DeMarco B. and Jin D. S., Science, 285 (1999) 1703.

[2] Schreck F. et al., Phys. Rev. Lett., 87 (2001) 080403.

[3] Truscott A. G. et al., Science, 291 (2001) 2570.

[4] Granade S. R., Gehm M. E., O'Hara K. M. and Thomas J. E., Phys. Rev. Lett., 88 (2002) 120405.

[5] Hadzibabic Z. et al., Phys. Rev. Lett., 88 (2002) 160401.

[6] Roati G., Riboli F., Modugno G. and Inguscio M., Phys. Rev. Lett., 89 (2002) 150403.

[7] Timmermans E., Phys. Rev. Lett., 87 (2001) 240403.

[8] Carr L. D., Shlyapnikov G. V. and Castin Y., Phys. Rev. Lett., 92 (2004) 150404.

[9] Jochim S. et al., Science, 302 (2003) 2101.

[10] Regal C. A., Greiner M. and Jin D. S., Phys. Rev. Lett., 92 (2004) 040403.

[11] Zwierlein M. W. et al., Phys. Rev. Lett., 92 (2004) 120403.

[12] Kinast J. et al., Phys. Rev. Lett, 92 (2004) 150402.

[13] Bourdel T. et al., Phys. Rev. Lett., 93 (2004) 050401.

[14] Chin C. et al., Science, 305 (2004) 1128.

[15] Timmermans E. and Côté R., Phys. Rev. Lett., 80 (1998) 3419.

[16] Lewenstein M., Cirac J. I. and Zoller P., Phys. Rev. A, 51 (1995) 4617.

[17] Geist W., You L. and Kennedy T. A. B., Phys. Rev. A, 59 (1999) 1500.

[18] Crescimanno M., Kaoy C. G. and Peterson R., Phys. Rev. A, 61 (2000) 053602.

[19] Holland M. J., Demarco B. and Jin D. S., Phys. Rev. A, 61 (2000) 053610.

[20] Papenbrock T., Salgueiro A. N. and Weidenmüller H. A., Phys. Rev. A, 65 (2002) $043601 ; 66$ (2002) 025603.

[21] Geist W. and Kennedy T. A. B., Phys. Rev. A, 65 (2002) 063617.

[22] Wouters M., Tempere J. and Devreese J. T., Phys. Rev. A, 66 (2002) 043414.

[23] Carr L. D., Bourdel T. and Castin Y., Phys. Rev. A, 69 (2004) 033603.

[24] Gardiner C., Quantum Noise (Springer-Verlag, Berlin) 1991.

[25] Carmichael H. J., Statistical Methods in Quantum Optics, Vol. 1 (Springer-Verlag, Berlin) 1999.

[26] Luiten O. J., Reynolds M. W. and Walrawen J. T. M., Phys. Rev. A, 53 (1996) 381.

[27] Holland M., Williams J. and Cooper J., Phys. Rev. A, 55 (1997) 3670.

[28] Elizalde E. et al., Zeta Regularization Techniques with Applications (World Scientific, Singapore) 1994. 